\title{
Delayed presentation of diaphragmatic hernia in a young and fit patient requiring emergency management
}

\author{
S.M. Tuladhar ${ }^{1}$ V.K. Sharma ${ }^{2}$
}

Departments of Surgery, National Academy of Medical Sciences, Kathmandu, Nepal

Diaphragmatic hernia, when they do present in adults, can manifest as a life threatening complication. Symptoms might initially be minimal in young and fit patients. They can also easily mimic complications like peptic ulcer perforation or tension pneumothorax and early pathophysiological correlation is required to reach an early diagnosis and treatment.

\section{Case History, Investigations and Treatment}

A 22 year old male presented with sudden upper abdominal pain and vomiting in the last 24 hours.

He was a non-smoker and non-alcoholic with a past history of acid peptic disease. Clinical findings of generalized tenderness with guarding and board like rigidity, rebound tenderness initially suggested his diagnosis of peptic ulcer perforation.

However, the chest X-ray also showed, along with gas under the right hemi-diaphragm, a hugely dilated stomach with a large fundal gas shadow. An unexpected feature was a pneumothorax on the left side with trachea and heart pushed to the right (figure 1).

Although with no respiratory symptoms initially, whilst in the emergency, he became progressively breathless and hypotensive. Aleft intercostal chest drain was quickly placed and intravenous fluids, oxygen and antibiotics were given as initial measures.

Intra-operatively, part of the hugely dilated stomach, the spleen and the splenic flexure of the large bowel was herniated into the left thoracic cavity. There was also volvolus of the stomach and a $2 \mathrm{~mm} \times 2 \mathrm{~mm}$

\section{Correspondences: S.M. Tuladhar}

E-mail: samptuladhar@yahoo.com posterior wall perforation. The left lung was collapsed and hypoplastic.

The volvolus was untwisted, the viability of the stomach was ascertained, the posterior wall perforation and finally the diaphragm was repaired. Splenectomy was also done to provide more space for the reduced stomach and colon contents. Finally, a feeding jejunostomy was also created.

Post-operatively, the patient was placed under intensive care with full cardiac and respiratory support.

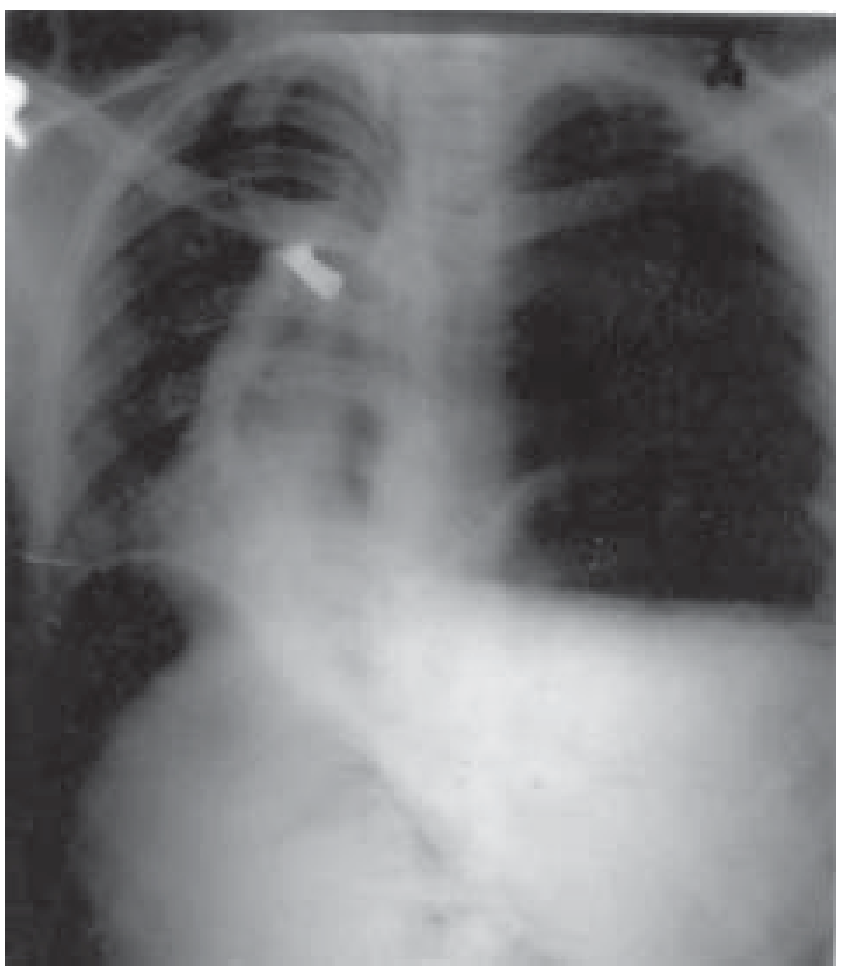


S.M. Tuladhar et al, Delayed presentation of diaphragmatic hernia in a young

\section{Discussion}

Our case was a Bochdalek's hernia which is a congenital posterolateral diaphragmatic hernia - an entity detected in adults usually only when they present with complications. ${ }^{1}$

Among diaphragmatic hernias, sliding hiatal hernias are the most common in adults. ${ }^{2}$ The Morgagni hernias and Bochdalek hernias are less common. Traumatic hernias can also occur after penetrating or blunt diaphragmatic rupture.

Respiratory failure due to congenital diaphragmatic herniation is a well-described entity in infants. ${ }^{3}$ Although not commonly seen in adults, when they do present, it may present as acute gastric dilatation. ${ }^{3}$ A herniated stomach can also twist giving rise to a volvolus. ${ }^{4}$ Strangulation due to a compromise in blood supply can lead to gangrenous changes. ${ }^{5}$ The patient may then present with clinical features of bowel perforation $^{6}$ and a misdiagnosis as peptic ulcer perforation could be done. It can also precipitate tension pneumothorax and present as an emergency life threatening respiratory failure. ${ }^{3}$ These emergency presentations could be lethal to the patient. ${ }^{6}$

Our case presented 24 hours after his symptoms began and, on first impression, was assumed to be duodenal ulcer perforation considering the nature of the history and features of generalized peritonitis. He had no chest symptoms initially. A concomitant feature of pneumothorax led us to think of the pathophysiology and take measures as early as possible.

\section{Conclusion}

Diaphragmatic herniation, when present in adults, usually manifests as complications. There may be initially minimal abdominal or respiratory complaints especially in a young and otherwise healthy patient. Peritonitis due to perforation and tension pneumothorax can be misdiagnosed if the pathophysiology is not closely correlated.

Once diagnosed by thorough careful examination and investigations, all possible cardiorespiratory parameters should be closely monitored and a joint team effort under the most optimal conditions will play a crucial role for a successful outcome.

\section{References}

1. I.D.Karanikas, S.S.Dendrinos, T.D.Liakakos et al Complications of congenital posterolateral diaphragmatic hernia in the adult. Report of two cases and literature review. J Cardiovasc Surg (Torino) 1994 Dec; 35(6):555-8

2. J. Moreaux, Diaphragmatic hernias in adults. Rev Prat 1997 Feb 1;47(3):277-81

3. N. Berkman, Z. Simon, Y. Almog, et al Acute gastric dilatation causing respiratory failure and "tension pneumothorax" in an elderly women with a diaphragmatic hernia. Chest 1993 Jul;104(1):317-8

4. I. Takanami, N. Morota, J. Shikata A case report of Bochdalek's hernia with volvulus of the stomach in an adult Nippon Kyobu Geka Gakkai Zasshi 1990 Sep;38(9):148891

5. A.N. Ghanem, T.S. Chankun, Brooks total gastric gangrene complicating adult Bochdalek hernia.PL Br J Surg 1987 Sep;74(9):779

6. P.P.Chui, C.T. Tan Sudden death due to incarcerated Bochdalek hernia in an adult. Ann Acad Med Singapore 1993 Jan;22(1):57-60 